Review

\title{
Prevalence and Incidence of Low Back Pain in the Kingdom of Saudi Arabia: A Systematic Review
}

\author{
Mai A. Aldera ${ }^{1,2, *}$, Caroline M. Alexander ${ }^{3}$, Alison H. McGregor ${ }^{1}$ \\ ${ }^{1}$ Faculty of Medicine, Department of Surgery and Cancer, MSK Lab, Imperial College Healthcare NHS Trust, Charing Cross Hospital, \\ Fulham Palace Road, London W6 8RF, UK \\ ${ }^{2}$ Department of Rehabilitation, King Saud University, Riyadh 11451, Saudi Arabia \\ ${ }^{3}$ Department of Therapies, Imperial College Healthcare NHS Trust, Charing Cross Hospital, Fulham Palace Road, London W6 8RF, UK
}

\section{ARTICLE INFO}

\section{Article History}

Received 07 November 2019

Accepted 08 April 2020

Keywords

Low back pain

Kingdom of Saudi Arabia

prevalence and incidence

\section{ABSTRACT}

Study Design: A systematic review.

Objective: To identify published studies that assess the prevalence and incidence of Low Back Pain (LBP) in the Saudi Arabian population.

Methods: Six electronic databases were searched for articles published between January 1995 and December 2018 . Crosssectional or cohort studies were included if they were conducted in the KSA and focused on the prevalence or incidence of LBP in adults. Case-control and retrospective studies were excluded. Studies were also excluded if they did not meet the quality criteria set out by the Joanna Briggs Institute (JBI) assessment or had a high or medium risk of bias according to the criteria proposed by Hoy et al. One independent reviewer (MAA) verified that the studies met the inclusion criteria, and three independent reviewers (MAA, AHM, CMA) assessed the quality of the studies and extracted their relevant characteristics. All the studies were assessed for quality using the JBI assessment and were assessed for risk of bias according to the Hoy et al. approach.

Results: The initial search identified 158 papers; five studies met the inclusion criteria. The nature of the findings meant no meta-analysis could be performed; therefore, a narrative summary was generated to discuss the findings. The prevalence of LBP in different professional groups within a working-age group ranged between $64 \%$ and $89 \%$.

Discussion: The prevalence of LBP in the KSA has only been examined within specific professional groups, which limits the ability to generalize the finding. The review clarifies the need for further quality epidemiological studies to identify the prevalence of LBP in the general population. Many of the issues identified are problems related to occupational risk of LBP. The implication therefore is that these occupational factors need to be assessed so that risk factors for LBP among employees in KSA can be modified.

\section{KEY POINTS}

1. The prevalence of LBP in the KSA has only been examined within specific professional groups.

2. The included studies link the prevalence of LBP to numbers of occupational-risk factors such as; participants' specialities, years of experience and working load.

3. The prevalence of LBP is more common in female employee.

4. This review outlines the need for further epidemiology studies of the general population in the KSA.

(C) 2020 The Authors. Published by Atlantis Press International B.V. This is an open access article distributed under the CC BY-NC 4.0 license (http://creativecommons.org/licenses/by-nc/4.0/).

\section{INTRODUCTION}

The Kingdom of Saudi Arabia's (KSA) experience of rapid socioeconomic change, had given rise to new public health challenges and extensive health reforms [1-3]. The 2017 Lancet review of the prevalence of Low Back Pain (LBP) in the adult general population concluded that prevalence was greater in high-income countries

"Corresponding author. Email: maldera@ksu.edu.sa

Data availability statement: The data that support the findings of this study are available from the corresponding author (MAA), upon reasonable request.
(30\%) than in low-income countries (18.2\%) and more common in females than males [4]. Thus, LBP has been recognized by governments as a major public health issue and a serious challenge for healthcare systems, leading to the declaration of a 'call for action' $[5,6]$.

The prevalence and incidence of LBP in the Saudi Arabian general population, and the factors associated with LBP are not clear and they vary in the literature. Most of the findings are presented in secondary analyses and are derived from studies evaluating work-related musculoskeletal (MSK) problems. However, with this growing evidence base it is now time to bring that literature 
together to inform our understanding and help judge its importance in relation to health policy in line with the new health reform of the Saudi Arabian 2030 vision, and to inform future research.

\section{AIM}

This systematic review sought to identify the prevalence and incidence of LBP in the Saudi Arabian population.

\section{METHODS}

A systematic review was conducted in line with the Preferred Reporting Items for Systematic Review and Meta-analysis (PRISMA) statement [7].

\subsection{Search Strategy and Selection}

The search strategy identified relevant studies by means of an online literature search using the following databases: Medline, PubMed, Cinahl, Embase, Cochrane, and Pedro. Additionally, data from the Saudi Arabian Ministry of Health and the Saudi Arabian General Authority for Statistics were searched.

The search terms used were: low back pain OR lower back pain OR a backache OR spondylosis OR lumbago OR coccyx OR mechanical low back pain OR non-specific low back pain AND Kingdom of Saudi Arabia OR Saudi Arabian OR KSA OR SA AND prevalence OR incidence.

\subsection{Study Criteria}

Studies were included if they met the following criteria: the paper included adults who were complaining of acute or chronic LBP; were cross-sectional or cohort studies published in peer-reviewed journals between January 1990 and December 2018. The publication dates were limited to start at 1990 to take into consideration the growth of the Saudi Arabian population since that time. Exclusion criteria were: studies involving people under 18 years of age; studies in which the primary focus of the study was not LBP, studies of pregnant women, drug trials, case-control and retrospective studies. Also, studies were excluded if they did not meet the quality criteria according to the Joanna Briggs Institute (JBI) assessment (http://joannabriggs.org) [8] and studies with high or medium risk of bias according to the Hoy et al. [9] criteria.

\subsection{Quality Assessment and Risk of Bias Assessment}

The methodological quality of the studies was evaluated using a specific tool developed for assessing the quality of prevalence papers. The JBI prevalence tool (http://joannabriggs.org) [8] defines sufficient quality by affirmative answers to 10 questions. The questions ask about different facets of quality; for example, one question is "Were study participants recruited appropriately?" The paper is scored by answering the questions with yes, no, unclear or not applicable.
Yes, suggests high quality, and no suggests poor quality. To be included in the review, six questions needed to score 'yes'.

The risk of bias was evaluated using criteria developed by Hoy et al. [9]. This has previously been used in studies assessing the prevalence of LBP [10,11]. An example of a question is "Was the likelihood of non-response bias minimal?" The response rate of the study must be $75 \%$ or above to score yes. The questions are scored by yes and no answers indicating a low or high risk of bias respectively. The overall result concludes that a study has a low risk of bias if the answer to 0-3 questions is no, moderate if the answer to 4-6 questions is no and high if the answer to 7 or more question is no.

\subsection{Data Extraction}

All references were exported to Mendeley reference manger and duplicates were removed. The researcher (MAA) screened all of the studies that were identified by this search by title and abstract, and those that did not meet the inclusion criteria were excluded from the review. The remaining studies were retrieved in full text and reviewed to assess the quality and risk of bias by three independent researchers (MAA, AHM and CMA), any disagreements regarding inclusion or exclusion on the full text were resolved by discussion between the researchers.

A standardized data-extraction sheet was developed, and the data was extracted from five included studies by three independent researchers (MAA, AHM and CMA) as shown in Table 1.

\section{RESULTS}

Of the 198 references identified in the initial search, 156 duplicates were removed. The remaining 44 studies were screened for eligibility. Twenty-six studies were removed as shown in Figure 1 because they did not meet the inclusion criteria, 21 studies remained and were subjected to the assessment of quality and risk of bias.

Thirteen of these 18 studies failed to meet the JBI criteria and were therefore excluded. The risk of bias tool indicated that six papers had a high risk of bias, seven papers had a moderate risk of bias and five studies had a low risk of bias. The studies that fulfilled the criteria for reporting prevalence were the same studies deemed to have a low risk of bias. Five studies [12-15] met the inclusion criteria and these studies are summarized in Table 1.

The nature of the findings limited our ability to perform a meta-analysis. For this reason, we generated a narrative summary of the findings. The five studies that met the inclusion criteria and showed a high level of quality and a low risk of bias, were all conducted in Saudi Arabia between 2013 and 2018.

\subsection{Overall Prevalence}

In this review, the included studies indicated that the prevalence of LBP may be associated with three socio-demographic factors; occupation-related problems, age, and gender. Overall LBP prevalence in the included studies was between $63.8 \%$ and $89 \%$, as demonstrated in Figure 2. The following subsections discuss the findings of the included studies in depth. 


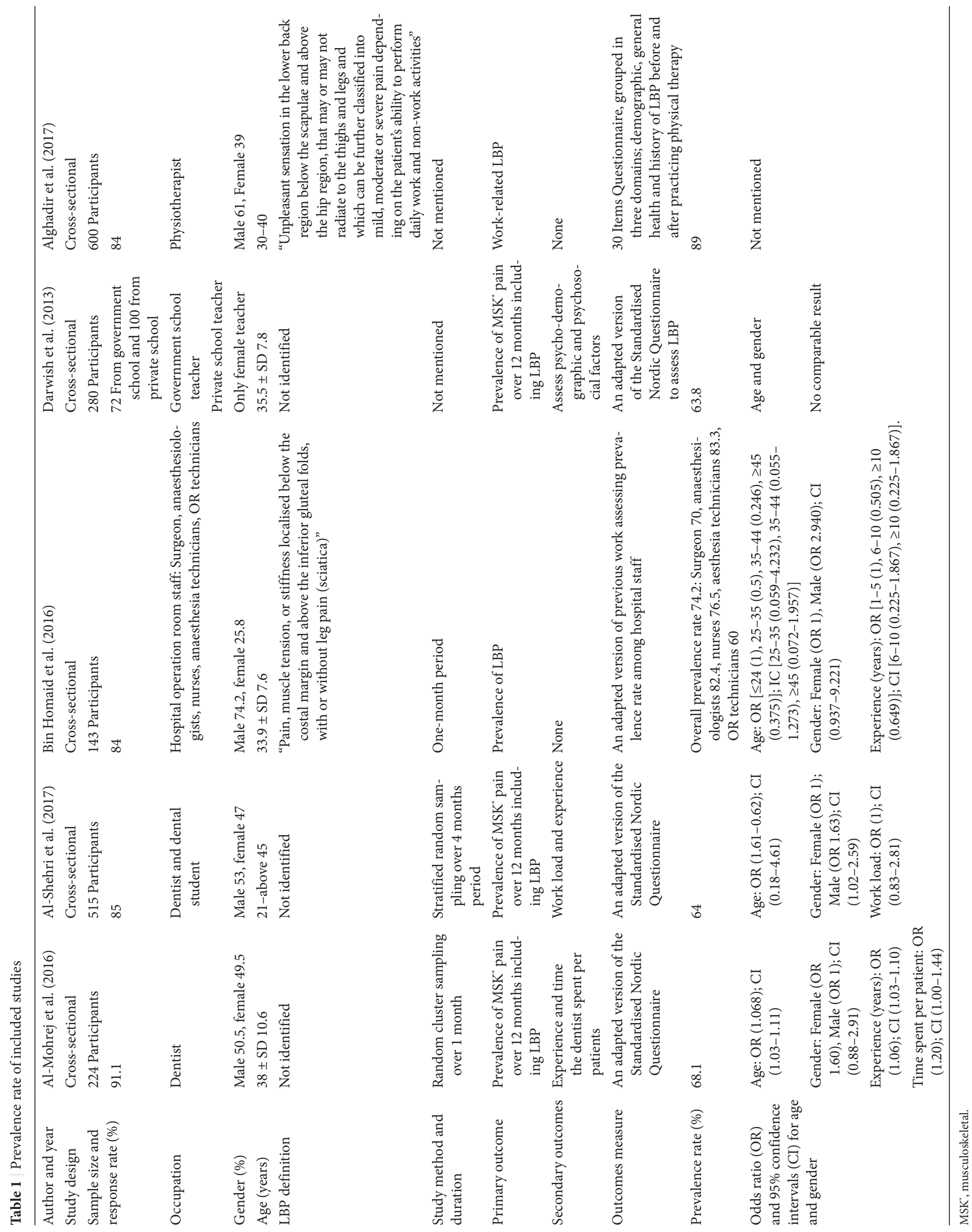




\section{The prevalence of low back pain in Saudi Arabia}

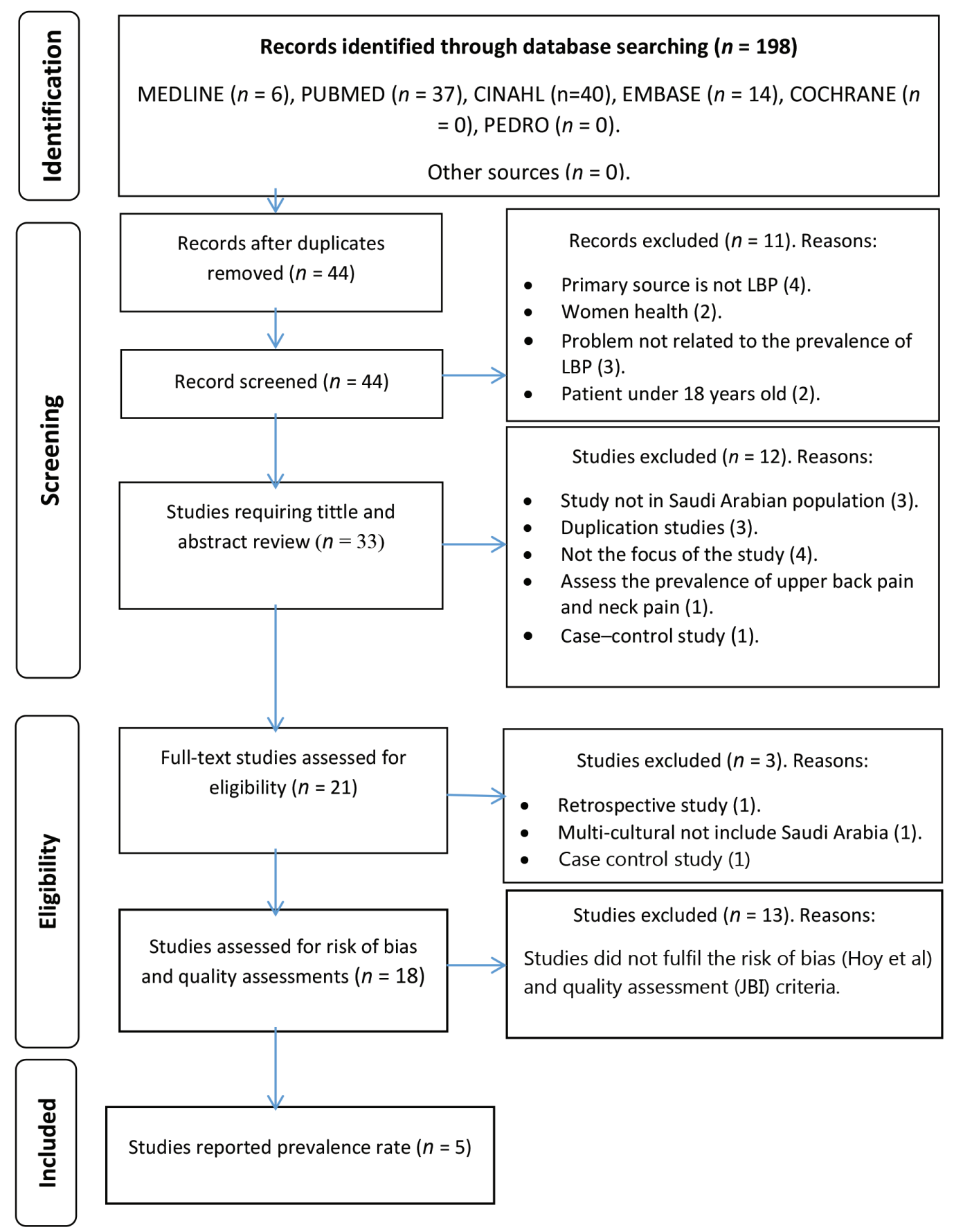

Figure $1 \mid$ PRISMA flow chart of the study selection process.

\subsection{Socio-demographic Factors}

\subsubsection{Occupational related risk factor for LBP in the KSA}

The primary aim of the included studies was to assess the prevalence of MSK pain including LBP problems among different disciplines. Overall LBP prevalence in the included studies was between $63.8 \%$ and $89 \%$. The highest prevalence percentage (89\%) reported was for physiotherapists in KSA [16], and teachers reported the lowest percentage (63.8\%). Two studies reporting on LBP in dentists identified similar prevalence percentages (64\% and 68\%) $[12,13]$, respectively. Finally, one study of Hospital Operation Room (HOR) staff (including members from different disciplines such as surgeons, anaesthesiologists, nurses, anaesthesia technicians, and operation room technicians) reported a prevalence of $74.2 \%$ [14].

In this review, the prevalence of LBP was associated with three factors related to occupational risk; speciality of the participants, years of experience and length of working hours. 


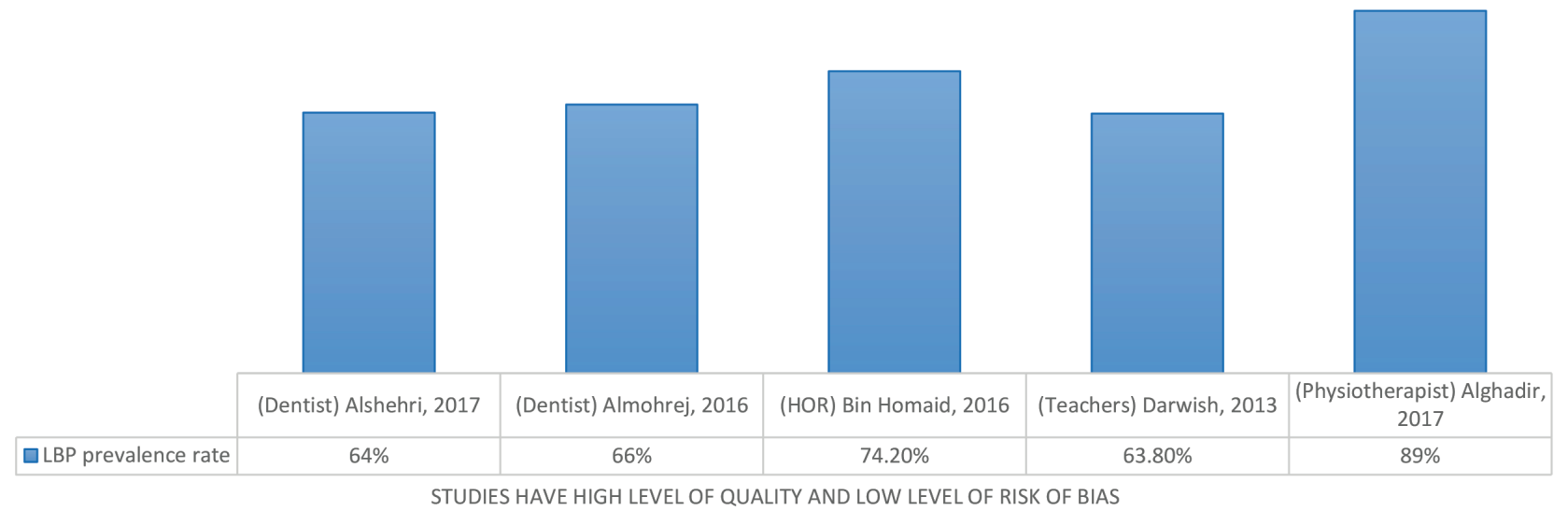

Figure 2 The prevalence of LBP in Saudi Arabian population.

Low back pain prevalence was found to be associated with participant speciality. For example, a study of physiotherapists conducted by Alghadir et al. [16] reported that the prevalence of the problem varied in terms of severity and location according to sub-speciality, whereby it was higher in neurology specialists (71\%) than in orthopaedic specialists (30\%). This finding was in line with the study of HOR staff conducted by Bin Homaid et al. [14], which reported an overall prevalence rate of $74 \%$, but a higher prevalence among anaesthesiologists $(82.4 \%)$ than among nurses and surgeons $(76.5 \%$ and $70 \%$, respectively).

Years of experience and working load were reported in the majority of the included studies. While some of the studies found strong positive association such as among dentists $[12,13]$, teachers [15] and physiotherapists [16]. One study was found negative correlation [14].

The impact of severe LBP on participants was measured only by the cross-sectional study of physiotherapists [16], which found that severe LBP forced $11 \%$ of the therapists to decrease their working hours and $34 \%$ of them to change their work settings or take sick leave.

\subsubsection{Age}

The included studies focused on specific disciplines and participants were restricted to the working-age group. As shown in Table 1, the age of participants was between 20 and more than 40 years old $[38 \pm$ SD 10.6, 21-above 45, 33.9 \pm SD 7.6, $35.5 \pm$ SD 7.8, and 30-40 years] [12-16].

\subsubsection{Gender}

One of the studies included in this review focused on female teachers in Saudi Arabian government and private schools [15], but the gender ratio was varied in the other studies: two studies had an equal gender ratio, and in the other two studies, females accounted for $25 \%$ and $39 \%$ of the participants (see Table 1). Thus, to avoid risk of selection bias, the results of the review are concluded from two studies $[14,16]$.

\section{DISCUSSION}

The aim of this review was to identify the prevalence and incidence of LBP in the Kingdom of Saudi Arabia. There were no studies of incidence and among general population. Five studies met the inclusion criteria of our review and indicated that the prevalence of LBP in the Saudi Arabian population was between $64 \%$ and $89 \%$.

\subsection{Overall Prevalence}

Despite the fact that the prevalence of LBP has only been explored within specific occupational disciplines in KSA, they are similar to the prevalence within the general population of other Gulf Cooperation Countries, of which KSA is a part. These countries share a similar geographic location and socio-demographic characteristics with KSA. For example, the LBP prevalence in the general population of the United Arab Emirates is 64.6\% (95\% CI, 60.768.5; [17]). In Qatar, it is a little lower at 56.5\% (95\% CI, 54.2-58.8; [18]). In contrast, developed countries have been found to have lower prevalence than developing countries. For example, the population prevalence in the United Kingdom (UK) is 36.1\% [19] and in Canada is $28.7 \%$ [20]. However, the fact that the included studies were restricted to specific disciplines compromise the generalizability of the findings to the broader population, and further epidemiological studies of the general population in KSA are needed.

\subsection{Socio-demographic Factors}

\subsubsection{Occupational risk factor for $L B P$}

Occupational risk factor for LBP was found in all the included studies in terms of specialities, working load and years of experience. These risk factors have also been described in other nations. For example, two cross-sectional studies were performed in Kuwait, one among health care professionals from different specialities [21] and the other among physiotherapists [22]. They also describe risk in terms of speciality and working load. For example, they found an association between tasks such as lifting and transferring patients and LBP $(p=0.02)$. Furthermore, a recent systematic review from 
a mix of developed countries conclude that working load such as high intensity of physical activities, lifting, bending and twisting is one of the important risk factors for LBP [23]. Finally, a recent study of the employees from Bahrain University, found a positive correlation between increasing years of experience and prevalence of LBP [24].

\subsubsection{Age factor}

The age of the participants within the included papers fell into a working-age group. This concentration upon working people is in line with pooled results from publications dealing with prevalence from 28 countries including those from the middle-east [5]. Their study found that LBP was 2.5 times more prevalent in a working population. In addition, Fatoye's et al.'s [23] systematic review identified age as an important risk factor for LBP throughout the developed countries.

\subsubsection{Gender factor}

The included studies found that LBP was more common in female Saudi Arabian participants than in male. Indeed, LBP has been found to be more common in females than in males globally [25]. However, it should be noted that some of the disciplines reported upon were gender dominant. For example, it is reasonable to assume that school teachers in KSA are more frequently female than male.

\section{CONCLUSION}

To date, limited studies have been conducted with regards to the prevalence and incidence of LBP in the KSA. The prevalence of LBP in the general population has not only been unrecognized, but has also not been accurately determined as a result of poor-quality studies.

\section{LIMITATION}

\subsection{Limitations}

All but one of the studies were conducted among healthcare staff, and most commonly, the data was collected from the populations in Riyadh city, the capital of KSA $(n=3)$. Therefore, the results of these studies may not be transferable to the prevalence within the general population of the whole of the KSA.

\subsection{Implications for Practice and Policy}

The findings of this study have significant implications for occupational health practice in KSA. Many of the issues identified in this review were problems related to occupational-risk of LBP, such as years of experience, workload, and employee speciality. This needs further examination, but encourages change in practice and policy to reduce risk of LBP in more experienced clinicians who are subjected to higher loading. One way to address this is to improve awareness of back care and safe patient handling for healthcare professionals.

\subsection{Implication for Research}

Further epidemiological research is needed to identify the prevalence of LBP in the general population of KSA. When prevalence is more widely understood it might help drive change that could impact the levels of disability from this painful condition.

\section{CONFLICTS OF INTEREST}

The authors declare they have no conflicts of interest.

\section{AUTHORS' CONTRIBUTION}

MAA, CMA and AHM contributed in study conceptulization, investigation, methodology, validation, Visualization and writing (review and editing) the manuscript. MAA contributed in data curation, formal analysis, resources, software, project adminstration and writing (original draft) the manuscript. No funding acquisition was needed. AHM and CMA supervised the project.

\section{ACKNOWLEDGMENTS}

Mai A. Aldera acknowledges support and fund from the Saudi Arabian Ministry of Education, King Saud University, Riyadh, Kingdom of Saudi Arabia. Alison McGregor acknowledges support from the NIHR Imperial Biomedical Research Centre (BRC).

\section{REFERENCES}

[1] Almalki M, Fitzgerald F, Clark M. Healthcare system in Saudi Arabia: an overview. Eastern Mediterr Health J 2011;17;784-93.

[2] Al-Sharqi OZ, Abdullah MT. "Diagnosing” Saudi health reforms: is NHIS the right "prescription"? Int J Health Plann Manage 2013;28;308-19.

[3] Yusuf N. Private and public healthcare in Saudi Arabia: future challenges. Int J Bus Econ Dev 2014;22;114-18.

[4] Maher C, Underwood M, Buchbinder R. Non-specific low back pain. Lancet 2017;389;736-47.

[5] Hartvigsen J, Hancock MJ, Kongsted A, Louw Q, Ferreira ML, Genevay S, et al. What low back pain is and why we need to pay attention. Lancet 2018;391;2356-67.

[6] Buchbinder R, van Tulder M, Öberg B, Costa LM, Woolf A, Schoene M, et al. Low back pain: a call for action. Lancet 2018;391;2384-88.

[7] Moher D, Liberati A, Tetzlaff J, Altman DG. Preferred reporting items for systematic reviews and meta-analyses: the PRISMA statement. Ann Intern Med 2009;151;264-9.

[8] http://joannabriggs.org/assets/docs/sumari/ReviewersManual_ The-Systematic-Review-of-Prevalence-and-Incidence-Data.pdf

[9] Hoy D, Brooks P, Woolf A, Blyth F, March L, Bain C, et al. Assessing risk of bias in prevalence studies: modification of an existing tool and evidence of interrater agreement. J Clin Epidemiol 2012;65;934-9.

[10] Overaas CK, Johansson MS, de Campos TF, Ferreira ML, Natvig B, Mork PJ, et al. Prevalence and pattern of co-occurring musculoskeletal pain and its association with back-related disability 
among people with persistent low back pain: protocol for a systematic review and meta-analysis. Syst Rev 2017;6;258.

[11] Edwards J, Hayden J, Asbridge M, Gregoire B, Magee K. Prevalence of low back pain in emergency settings: a systematic review and meta-analysis. BMC Musculoskelet Disord 2017;18;143.

[12] Al-Shehri Z, Al Zoughool M. Prevalence and risk factors of musculoskeletal symptoms among dental students and dental practitioners in Riyadh City, Saudi Arabia. Arch Environ Occup Health 2017;73;56-63.

[13] Al-Mohrej OA, AlShaalan NS, Al-Bani WM, Masuadi EM, Almodaimegh HS. Prevalence of musculoskeletal pain of the neck, upper extremities and lower back among dental practitioners working in Riyadh, Saudi Arabia: a cross-sectional study. BMJ Open 2016;6;e011100.

[14] Bin Homaid M, Abdelmoety D, Alshareef W, Alghamdi A, Alhozali F, Alfahmi N, et al. Prevalence and risk factors of low back pain among operation room staff at a Tertiary Care Center, Makkah, Saudi Arabia: a cross-sectional study. Ann Occup Environ Med 2016;28;1.

[15] Darwish MA, Al-Zuhair SZ. Musculoskeletal pain disorders among secondary school Saudi female teachers. Pain Res Treat $2013 ; 2013 ; 878570$.

[16] Alghadir A, Zafar H, Iqbal ZA, Al-Eisa E. Work-related low back pain among physical therapists in Riyadh, Saudi Arabia. Workplace Health Saf 2017;65;337-45.

[17] Bener A, El-Rufaie OF, Siyam A, Abuzeid MSO, Toth F, Lovasz G. Epidemiology of low back pain in the United Arab Emirates. APLAR J Rheumatol 2004;7;189-95.
[18] Bener A, Dafeeah EE, Alnaqbi K. Prevalence and correlates of low back pain in primary care: what are the contributing factors in a rapidly developing country. Asian Spine J 2014;3;227-36.

[19] Raspe H, Matthis C, Croft P, O’Neill T. Variation in back pain between countries: the example of Britain and Germany. Spine (Phila Pa 1976) 2004;29;1017-21.

[20] Cassidy JD, Carroll LJ, Côté P. The Saskatchewan health and back pain survey: the prevalence of low back pain and related disability in Saskatchewan adults. Spine (Phila Pa 1976) $1998 ; 23 ; 1860-7$.

[21] Landry MD, Raman SR, Sulway C, Golightly YM, Hamdan E. Prevalence and risk factors associated with low back pain among health care providers in a Kuwait hospital. Spine (Phila Pa 1976) 2008;33;539-45.

[22] Shehab D, Al-Jarallah K, Moussa MAA, Adham N. Prevalence of low back pain among physical therapists in Kuwait. Med Princ Pract 2003;12;224-30.

[23] Fatoye F, Gebrye T, Odeyemi I. Real-world incidence and prevalence of low back pain using routinely collected data. Rheumatol Int 2019;39;619-26.

[24] Tantawy S. Work-related musculoskeletal symptoms among employees with different tasks: Ahlia University case study. Biomed Res 2019;30;1-6.

[25] Bento TPF, Genebra CVDS, Maciel NM, Cornelio GP, Simeão SFAP, de Vitta A. Low back pain and some associated factors: is there any difference between genders? Braz J Phys Ther 2020;24;79-87. 